\title{
Desarrollo profesional y autoeficacia docente del profesor universitario, Universidad Nacional Hermilio Valdizán - Huánuco 2013
}

Professional development and efficacy of teaching university professor, University

\author{
National Hermilio Valdizán - Huánuco 2013 \\ Ewer Portocarrero M. ${ }^{1}$ \\ Universidad Nacional Hermilio Valdizan, Huánuco Perú \\ (RECIBIDO 25/03/2014, ACEPTADO 27/05/2014)
}

\begin{abstract}
RESUMEN
Objetivo. Determinar el grado de relación entre el desarrollo profesional y la autoeficacia docente. Métodos. Se llevó a cabo un estudio correlacional, con 125 docentes de la Universidad Nacional Hermilio Valdizán, Huánuco, durante el período 2013. Se utilizó una ficha de análisis documental y una escala de autoeficacia. Para el análisis inferencial se utilizó el coeficiente de correlación de Pearson. Resultados. En cuanto al desarrollo profesional, $78.4 \%$ de los docentes alcanzaron el grado académico de magister y $52.0 \%$ de ellos fueron de dedicación exclusiva. El $76.0 \%$ tuvo autoeficacia alta y el $59.2 \%$, alta frecuencia de la misma. Se encontró relación significativa y positiva entre el desarrollo profesional y la autoeficacia docente $(\mathrm{r}=0.64 ; \mathrm{p} \leq 0.000)$ y en las dimensiones planificación de la enseñanza $(\mathrm{r}=0.64 ; \mathrm{p} \leq 0.000)$, implicación de los alumnos en el aprendizaje $(\mathrm{r}=0.64 ; \mathrm{p} \leq 0.000)$, interacción y creación de un clima positivo en el aula $(\mathrm{r}=0.63 ; \mathrm{p} \leq 0.000)$ y evaluación del aprendizaje $(r=0.62 ; p \leq 0.000)$. Conclusiones. Existe relación entre el desarrollo profesional y la autoeficacia docente del profesor universitario de la Universidad Nacional Hermilio Valdizán de Huánuco.
\end{abstract}

Palabras clave: autoeficacia, desarrollo profesional, docentes.

\begin{abstract}
Objective. To determine the degree of relationship between professional development and teacher self-efficacy. Methods. They conducted a correlational study with 125 teachers of the National University Hermilio Valdizan, Huanuco during 2013. We used a form of documentary analysis and self-efficacy scale. For inferential analysis we used the Pearson correlation coefficient. Results. As for the professional development of teachers $78.4 \%$ achieved academic master's degree and $52.0 \%$ were exclusive dedication. The $76.0 \%$ had high self-efficacy and high frequency of $59.2 \%$ the same. They found a significant positive relationship between professional development and teacher self-efficacy $(r=0.64, p \leq$
\end{abstract}

1 Docente Principal de la Facultad de Educación de la Universidad Nacional Hermilio Valdizán, Huánuco, Perú. E-mail: eportocarrero85@hotmail.com 
DESARROLLO PROFESIONAL Y AUTOEFICACIA DOCENTE DEL PROFESOR UNIVERSITARIO...

$0.000)$ and planning dimensions of teaching $(r=0.64, p \leq 0.000)$, involvement of students in learning $(\mathrm{r}=0.64, \mathrm{p} \leq 0.000)$, interaction and creation of a positive climate in the classroom $(r=0.63, p \leq 0.000)$ and assessment of learning $(r=0.62, p \leq 0.000)$. Conclusions. There is relationship between professional development and teacher self-efficacy of university professors from the National University of Huanuco Valdizan Hermilio.

Keywords: Self-efficacy, Professional Development, Teachers.

\section{INTRODUCCIÓN}

El principal impulsor de las variaciones en el aprendizaje universitario es la calidad de los docentes, según algunas investigaciones, y es por esto que una acción fundamental para mejorar los resultados del sistema educativo es fortalecer el desarrollo profesional de ellos (National board for professional teaching standards, 2005). Los países que han mejorado sus niveles educacionales a gran velocidad plantean que uno de los puntos claves para lograrlo es desarrollar a los docentes hasta convertirlos en instructores eficientes (Barber y Mourshed, 2008).

Es así que las expectativas y los retos que recaen en la profesión docente incrementan cada vez más el nivel de exigencia. Tal y como señala Gómez (2000, p. 19), "nunca hasta ahora el docente había estado sometido a demandas tan complejas, intensas y contradictorias, y este nuevo escenario afecta a nuestra eficacia profesional tanto como a nuestro equilibrio y desarrollo humano".

En su investigación, Herrera (2011) encontró que las condiciones de la eficacia del docente predicen los niveles de logro académico para él y el de sus estudiantes, independientemente de sus habilidades. El bajo sentido de la eficacia para satisfacer las demandas académicas afecta a los estudiantes positiva y/o negativamente.

Desde la teoría social cognitiva se define la autoeficacia como las "creencias en las propias capacidades para organizar y ejecutar los cursos de acción requeridos que producirán determinados logros o resultados" (Bandura, 1997) y se considera de vital importancia en el control de los elementos del entorno. El concepto hace referencia a la propia capacidad del individuo para organizar y ejecutar los cursos de acción requeridos para manejar situaciones futuras.

Bandura (1995) señala que los efectos de las creencias de eficacia sobre los procesos cognitivos se pueden explicar de la manera siguiente: mucho del comportamiento intencional del ser humano está regulado por metas significativas para la persona; el planteamiento de metas está afectado por la evaluación de las capacidades personales. Por lo tanto, cuanta más alta sea percibida la autoeficacia personal, más alto será el nivel de las metas que las personas se impongan.

La autoeficacia del profesor se considera importante dado el impacto que posee sobre los estudiantes, ya que existe una relación positiva entre este constructo y las conductas del alumnado en la sala de clases (Kagan, 1990 citado en González, 1999). 
Asimismo, la autoeficacia docente se enmarca en lo que se conoce como "creencias pedagógicas del profesorado", entendidas como las concepciones o teorías implícitas del docente que generan una disposición a actuar de una manera determinada. Woolfolk y Murphy (2001, citado en Chacón, 2006) sugieren que una vez que la percepción de la autoeficacia se establece en el sistema de creencias de autoeficacia constituye un factor decisivo en el logro de metas que cada quien se propone. Si las personas creen que no tienen poder para producir resultados, no harán el intento para hacer que esto suceda.

Este tipo de creencias influyen en todos los aspectos de la práctica docente, debido a la imposibilidad de desvincular lo que las personas se juzgan capaces de hacer y las acciones que, en función de ello, deciden emprender. Lo anterior sitúa la autoeficacia en una posición preferente en relación con otras variables del profesorado (Prieto, 2005). Al respecto, Chacón (2006) plantea que la autoeficacia percibida afecta el ambiente de aprendizaje propiciado por el docente y sus acciones, así como el esfuerzo y la persistencia para lograr el aprendizaje de los estudiantes.

Según Chacón (2006), las creencias de autoeficacia que los profesores perciben en relación con sus capacidades para facilitar el aprendizaje de los estudiantes constituyen una fuente fundamental de información que permite interpretar las acciones del docente en el aula. La eficacia del docente se considera como el principal predictor del comportamiento del docente, en relación con el esfuerzo y persistencia que dedica a sus actividades de enseñanza y su compromiso para apoyar y mejorar los aprendizajes de sus alumnos. Por otra parte, estudios tales como los realizados por Prieto (2005), han demostrado que profesores con un elevado sentimiento de autoeficacia muestran mayor apertura a nuevas ideas, están más dispuestos a probar métodos innovadores, así es que ellos adaptan mejor sus clases, dedican más tiempo y energía a los alumnos que se esfuerzan en su aprendizaje, manifiestan gran entusiasmo por la enseñanza y se encuentran más comprometidos en su profesión.

Con base en lo expuesto, se decidió realizar esta investigación con el objetivo de determinar el grado de relación entre el desarrollo profesional y la autoeficacia docente del profesor universitario de la Universidad Nacional Hermilio Valdizán.

\section{MÉTODO}

\section{Tipo de estudio y diseño}

Según el análisis y alcance de los resultados el estudio fue de tipo observacional, prospectivo, transversal y analítico.

El diseño que se utilizó en esta investigación fue el descriptivo correlacional, tal como se muestra a continuación: 
DESARROLLO PROFESIONAL Y AUTOEFICACIA DOCENTE DEL PROFESOR UNIVERSITARIO...

$0 \mathrm{x}$

M r

$0 \mathrm{y}$

Donde:

$\mathrm{M}=$ Conjunto de elementos que conforman la muestra

$0 \mathrm{x}=$ Información sobre la variable 1

0y $=$ Información sobre la variable 2

$\mathrm{r}=$ Grado de relación

\section{Población}

La población de estudio estuvo conformada por los profesores universitarios nombrados de la Universidad Nacional Hermilio Valdizán de Huánuco, que hicieron un total de 374 profesores, distribuida en las 14 facultades y 27 carreras profesionales. Asimismo, se tuvo en cuenta una muestra total de 125 docentes nombrados, seleccionados por muestreo no probabilístico.

\section{Procedimiento}

Se emplearon como instrumentos de recolección de datos una ficha de análisis documental donde se recabó información sobre el desarrollo profesional de los docentes en estudio, mediante los legajos personales que obran en la oficina de escalafón de la universidad. Por otro lado, para la variable autoeficacia docente se utilizó una escala de autoeficacia docente del profesor universitario, de Leonor Prieto Navarro (2005).

La medición cuantitativa del desarrollo profesional fue establecida mediante las puntuaciones por los indicadores de dedicación exclusiva, tiempo completo, estudios de maestría, doctorado, especialidad, diplomados y estudios concluidos de otra profesión. En cuanto a la autoeficacia docente del profesor universitario, se utilizaron las dimensiones de las estrategias didácticas para la planificación de la enseñanza, implicar activamente a los alumnos, favorecer la interacción en el aula y evaluar el aprendizaje con respuestas que fueron de 1 a 6 puntos.

\section{Análisis de datos}

En el análisis descriptivo de los datos se utilizaron las medidas de tendencia central y de dispersión y en el análisis inferencial se utilizó el coeficiente de correlación de Pearson para medir la relación entre variables cuantitativas. Se tuvo en cuenta una significación de 0.05. En el procesamiento de los datos se utilizó el paquete estadístico SPSS versión 19.0 para Windows. 
Ewer Portocarrero M.

\section{RESULTADOS}

Dentro de las características generales de los docentes en estudio, encontramos que el $33.6 \%$ (42 docentes) tuvo rango de edades de 45 a 53 y 54 a 62 años, cada una. Por otro lado, el $40.8 \%$ (51 docentes) alcanzó la categoría de asociado y el $30.4 \%$ (38 docentes) se encontraba con tiempo de servicio de 11 a 15 y 16 a 20 años, cada una (tabla 1).

En cuanto al desarrollo profesional, el 78.4\% (98 docentes) alcanzó el grado académico de maestro o magíster; el 52.0\% (65 docentes) fue de dedicación exclusiva; el $40.0 \%$ (50 docentes) tuvo estudios de diplomado; el $32.8 \%$ (41 docentes) fue de tiempo completo; el 27.2\% (34 docentes) contó con grado académico de doctor y el $20.0 \%$ (25 docentes) tuvo estudios concluidos de segunda especialización. Asimismo, los docentes en estudio alcanzaron otros diplomados en 5 últimos años, estudios concluidos de otra profesión (carrera), otros títulos de segunda especialización, otros grados académicos de maestro o magíster y estudios concluidos de posdoctorado (figura 1).

Respecto a la autoeficacia de los docentes en estudio, observamos que en general el $76.0 \%$ de los docentes tuvieron autoeficacia alta y el 59.2\% alta frecuencia de ella. Según las dimensiones, en planificación de la enseñanza el $74.4 \%$ tuvo alta autoeficacia y el 59.2\% fue con alta frecuencia; en implicación de los alumnos en el aprendizaje el $76.8 \%$ obtuvo alta autoeficacia y el $61.6 \%$ fue con alta frecuencia; en la interacción y creación de un clima positivo en el aula el $76.0 \%$ tuvo alta autoeficacia y el $60.0 \%$ fue con alta frecuencia; y en la evaluación del aprendizaje el $70.4 \%$ tuvo alta autoeficacia y el $59.2 \%$ fue con alta frecuencia (tabla 2).

En cuanto al análisis inferencial de los resultados, se encontró relación significativa entre el desarrollo profesional y la autoeficacia docente $(r=0.64 ; p \leq 0.000)$. Del mismo modo, se halló relación significativa entre desarrollo profesional y las dimensiones de autoeficacia en estrategias didácticas para la planificación de la enseñanza $(\mathrm{r}=0.64 ; \mathrm{p} \leq 0.000)$; estrategias didácticas para implicar activamente a los estudiantes $(\mathrm{r}=0.64 ; \mathrm{p} \leq 0.000)$; estrategias didácticas para favorecer la interacción en el aula $(\mathrm{r}=0.63 ; \mathrm{p} \leq 0.000)$ y estrategias didácticas para evaluar el aprendizaje ( $\mathrm{r}=0.62 ; \mathrm{p} \leq 0.000)$ (tabla 3$)$. Asimismo, según la dispersión de puntos se encontró que a mayores valores de las puntuaciones de la autoeficacia docente la variable desarrollo profesional adquiere también mayores valores (figura 2).

\section{DISCUSIÓN}

El docente universitario "se enfrenta hoy a una situación de cambio en la que se le solicita que asuma un rol diferente al hasta ahora asignado" (Rodríguez, 2002, pp. 53); no solamente debe ser un conocedor de la ciencia, un experto en técnicas y un investigador, sino que debe ser guía y supervisor de la formación científica del estudiante. 
Ser profesor no es un estatus que se adquiere a través de unas pruebas, sino una actividad que se ejerce en el acto de enseñar y que cada día se renueva y perfecciona. De ahí que se plantee como objetivo esencial del desarrollo profesional que el profesor se sitúe ante un proceso de formación continua para que cada día pueda mejorar la práctica. El profesor debe convertirse diariamente en un aprendiz, no se puede considerar que la condición de profesor se adquiera una vez superada la etapa de la formación inicial. Al contrario, el profesor se hace cada día en la medida que el proceso de formación continua le permite hacer su trabajo cada vez mejor, para lo cual tiene que mantener una constante actitud de aprendizaje. La formación durante el ejercicio profesional constituye una exigencia inseparable de la condición de profesor (Allinder, 1994).

En nuestra investigación, la relación entre la autoeficacia docente y el desarrollo profesional de los docentes participantes del estudio fue de 0.64 , significativa estadísticamente $(\mathrm{P} \leq 0.000)$. Esto quiere decir que la autoeficacia docente se relaciona directamente con el desarrollo profesional de los docentes. Lo mismo sucedió con las dimensiones de la autoeficacia docente.

$\mathrm{Al}$ respecto, en la actualidad existen suficientes pruebas sobre la influencia de la autoeficacia percibida en el desarrollo profesional. Las conclusiones de los estudios realizados sugieren que las creencias de eficacia ejercen una influencia directa sobre la toma de decisiones y el propio desempeño profesional. Una baja eficacia percibida podría restringir indebidamente los tipos de ocupaciones consideradas e influir sobre la ejecución y persistencia en el logro de la profesión seleccionada (Hackett, 1995); mientras que una alta autoeficacia percibida da lugar típicamente a una mayor motivación para emprender a niveles más altos de logro (Gibbons y Weingart, 2001).

Muy semejante es la investigación de Tschannen-Mora, Woolfolk y Hoy (1998), quienes plantean que "la autoeficacia docente se relaciona con el rendimiento, motivación, percepción de la eficacia en los alumnos y profesores, y su accionar en el aula. Al respecto, por ejemplo, un profesor con alta autoeficacia mostrará mayor apertura a las ideas nuevas, planificará mejor sus actividades y probará nuevas metodologías de enseñanza. Además, los docentes con adecuadas creencias de autoeficacia se encuentran motivados a enseñar, dedican más tiempo a los alumnos que les cuesta aprender y demuestran su compromiso por educar".

Aquellos profesores con alta autoeficacia emplean más tiempo en la clase para el desarrollo de actividades académicas, utilizan métodos instructivos más complejos, proporcionan más ayuda y orientación a los alumnos y elogian sus logros académicos (Skaalvik y Skaalvik, 2007; Wolters y Daugherty, 2007). Por el contrario, los profesores con baja autoeficacia dedican más tiempo de clase a tareas no académicas, retiran el apoyo a los alumnos cuando no alcanzan relativamente pronto los resultados previstos y critican los errores que estos cometen.

La autoeficacia tiene un papel vital en el ámbito académico. De acuerdo con estudios, se ha evidenciado que un buen desempeño académico no puede ser garantizado solo por los conocimientos y habilidad de los individuos. Las creencias de eficacia 
pueden determinar un desempeño diferente en dos personas con el mismo grado de habilidad. Esto se debe a que el éxito académico demanda procesos reguladores como la autoevaluación, el automonitoreo y el uso de estrategias metacognitivas de aprendizaje, procesos que son influidos positivamente por un alto grado de creencia en la propia capacidad o autoeficacia (Pajares, 2002).

\section{CONCLUSIONES}

Según los resultados derivados de la investigación realizada sobre este tema, los sentimientos de competencia y de eficacia personal no solo parecen influir en las expectativas de éxito como docentes, sino que tienen claras consecuencias sobre la motivación y el rendimiento de sus estudiantes (Skaalvik y Skaalvik, 2007; Wolters y Daugherty, 2007). A este respecto, enseñar a los profesores a ser más autoeficaces debería contribuir a que los alumnos mejoren sus capacidades para gestionar los recursos personales y contextuales vinculados a un aprendizaje de calidad y, como consecuencia, mejoren también su grado de motivación y sus resultados académicos.

Tschannen-Moran y WoolfoolK (2001) afirman que aquellos profesores con altas creencias de autoeficacia presentan mayor apertura a nuevas ideas, mayor disposición para probar nuevos métodos, mejor planificación y organización de sus clases y se muestran más entusiastas en el proceso de enseñanza.

Se sugiere a las instituciones de educación superior centrarse en programas de estudio que busquen formar profesores integrales, que incluyan dentro de las mallas curriculares contenidos, estrategias y técnicas más actuales y contextualizadas, especialmente en el desarrollo de la autoeficacia docente, desde el inicio de la instrucción hasta los últimos años. Además, el estudio abordado se convierte por sí mismo en una línea de investigación que potencia la posibilidad de profundizar al respecto en investigaciones posteriores.

En conclusión se encontró relación directa y positiva entre el desarrollo profesional y la autoeficacia docente del profesor universitario de la Universidad Nacional Hermilio Valdizán. Huánuco, 2013.

\section{REFERENCIAS BIBLIOGRÁFICAS}

Allinder, R. (1994) "The relationship between efficacy and the instructional practices of special education teachers and consultants". Teacher Education and Special Education, 17, 86-95.

Bandura, A. (1995) "Exercice of personal and collective efficacy". En: Bandura, A. (ed.) Self - efficacy in Changing Societies, EEUU: University of Cambridge.

Bandura, A. (1997) "Self-efficacy: The exercise of control”. New York, NY: Freeman.

Barber, M y Mourshed, M. (2008) "Cómo hicieron los sistemas educativos con mayor desempeño del mundo para alcanzar sus objetivos". Santiago: PREAL. 
DESARROLLO PROFESIONAL Y AUTOEFICACIA DOCENTE DEL PROFESOR UNIVERSITARIO...

Chacón, C. (2001) "Las creencias de autoeficacia: un aporte para la formación docente de inglés". Acción pedagógica, 15, 44-54.

Gibbons, D.E. y Weingart, LR. (2001) "Can I do it? Will I try? Personal efficacy, assigned goals, and performance norms as motivators of individual performance”. Journal of Applied Social Psychology, 31, 3, pp. 624-648.

Gómez, L. (2000) "Marco de intervención en la prevención del estrés y resolución de conflictos docentes". En L. GÓMEZ; J. CARRASCOSA (coords): Prevención del estrés profesional docente. Valencia, Generalitat Valenciana.

González, M. (1999) “Creencias, atribuciones y auto-eficacia en profesores de enseñanza básica municipalizada” (Tesis doctoral). Facultad de Educación, Pontificia Universidad Católica de Chile, Santiago, Chile.

Hackett, G. (1995) "Self-effi cacy in career choice and development", en Albert Bandura (Ed.) Self-effi cacy in changing societies (pp. 232-258), Nueva York: Cambridge University Press.

Herrera, M. (2011) “Autoeficacia del Docente Universitario". Centro de Orientación de la Facultad de Humanidades y Educación de la Universidad del Zulia. Maracaibo.

National Board for Professional Teaching Standards. Standards and National Board Certification. (2005) Disponible en: http://www.nbpts.org/standardsnbcert.cfin

Pajares, F. (2002) "Overview of Social Cognitive Theory and Self - Efficacy". EEUU: Emory University.

Prieto, L. (2005) "Las creencias de autoeficacia docente del profesorado universitario". (Tesis doctoral no publicada). Universidad Pontificia Comillas, Madrid.

Rodríguez, G. (2002) "El reto de enseñar hoy en la Universidad”. En V. Álvarez y A. Lázaro (Coods.), Calidad de las universidades y orientación universitaria (pp. 49-58). Málaga: Aljibe.

Skaalvik, E.M. y Skaalvik, S. (2001) "Dimensions of teacher self-efficacy and relations with strain factors, perceived collective teacher efficacy, and teacher burnout". Journal of Educational Psychology, 99, 611-625.

Tschannen-Moran, M. y Woolfolk, A. (2001) "Teacher efficacy: capturing an elusive construct". Teaching and Teacher Education, 17, 783-805.

Tschannen-Moran, M., Woolfolk, A. y Hoy, W.K. (1998) "Teacher efficacy: Its meaning and measure". Review of Educational Research, 68 (2), 202-248.

Wolters, C.A. y Daugherty, SG. (2007) "Goals structures and teachers' sense of efficacy: Their relation and association to teaching experience and academic level". Journal of Educational Psychology, 99, 181-193. 


\section{ANEXOS}

Tabla 1. Aspectos generales de los docentes de la Universidad Nacional Hermilio Valdizán. Huánuco, 2013.

\begin{tabular}{lcc}
\hline Aspectos generales & $\begin{array}{c}\text { Frecuencia } \\
\text { (n=125) }\end{array}$ & $\%$ \\
\hline \hline \multicolumn{1}{c}{ Edad en años } & & \\
36 a 44 & 24 & 19,2 \\
45 a 53 & 42 & 33,6 \\
54 a 62 & 42 & 33,6 \\
63 a 71 & 17 & 13,6 \\
$\quad$ Categoría & & \\
Auxiliar & 32 & 25,6 \\
Asociado & 51 & 40,8 \\
Principal & 42 & 33,6 \\
Tiempo de servicio & & \\
$\quad$ en años & & \\
1 a 5 & 2 & 1,6 \\
6 a 10 & 10 & 8 \\
11 a 15 & 38 & 30,4 \\
16 a 20 & 38 & 30,4 \\
21 a más & 37 & 29,6 \\
\hline
\end{tabular}

Fuente: Elaboración propia.

Tabla 2. Dimensiones de la autoeficacia de los docentes de la Universidad Nacional Hermilio Valdizán. Huánuco, 2013.

\begin{tabular}{lcccc}
\hline & \multicolumn{3}{c}{ Autoeficacia $\begin{array}{c}\text { Frecuencia de } \\
\text { autoeficacia }\end{array}$} \\
\cline { 2 - 5 } Dimensiones/Global & \multicolumn{4}{c}{ (n=125) } \\
\cline { 2 - 5 } & № & \% & № & \% \\
\hline \multicolumn{1}{c}{ Planificación de la } & & & & \\
enseñanza & & & & \\
Alta & 93 & 74,4 & 74 & 59,2 \\
Media & 14 & 11,2 & 22 & 17,6 \\
Baja & 18 & 14,4 & 29 & 23,2 \\
Implicación de los alumnos & & & & \\
$\quad$ en el aprendizaje & & & & \\
Alta & 96 & 76,8 & 77 & 61,6 \\
Media & 12 & 9,6 & 24 & 19,2 \\
Baja & 17 & 13,6 & 24 & 19,2 \\
Interacción y creación de un & & & & \\
clima positivo en el aula & & & & \\
Alta & 95 & 76,0 & 75 & 60,0 \\
Media & 15 & 12,0 & 29 & 23,2 \\
Baja & 15 & 12,0 & 21 & 16,8 \\
Evaluación del aprendizaje & & & & \\
Alta & 88 & 70,4 & 74 & 59,2 \\
Media & 17 & 13,6 & 27 & 21,6 \\
Baja & 20 & 16,0 & 24 & 19,2 \\
Autoeficacia docente del & & & & \\
$\quad$ profesor universitario & & & & \\
Alta & 95 & 76,0 & 74 & 59,2 \\
Media & 11 & 8,8 & 27 & 21,6 \\
Baja & 19 & 15,2 & 24 & 19,2 \\
\hline
\end{tabular}

Fuente: Elaboración propia. 
DESARROLLO PROFESIONAL Y AUTOEFICACIA DOCENTE DEL PROFESOR UNIVERSITARIO...

Tabla 3. Correlación entre las dimensiones de la autoeficacia y el desarrollo profesional de los docentes de la Universidad Nacional Hermilio Valdizán. Huánuco, 2013.

\begin{tabular}{|c|c|c|}
\hline \multirow{2}{*}{ Variables } & \multicolumn{2}{|c|}{ Desarrollo profesional } \\
\hline & $\begin{array}{c}\text { rde } \\
\text { Pearson }\end{array}$ & Significancia \\
\hline Planificación de la enseñanza & 0,64 & 0,000 \\
\hline $\begin{array}{l}\text { Implicación de los alumnos en el } \\
\text { aprendizaje }\end{array}$ & 0,64 & 0,000 \\
\hline $\begin{array}{l}\text { Interacción y creación de un clima } \\
\text { positivo en el aula }\end{array}$ & 0,63 & 0,000 \\
\hline Evaluación del aprendizaje & 0,62 & 0,000 \\
\hline $\begin{array}{l}\text { Autoeficacia docente del profesor } \\
\text { universitario }\end{array}$ & 0,64 & 0,000 \\
\hline
\end{tabular}

Fuente: Elaboración propia.

Figura 1. Desarrollo profesional de los docentes de la Universidad Nacional Hermilio Valdizán. Huánuco, 2013.

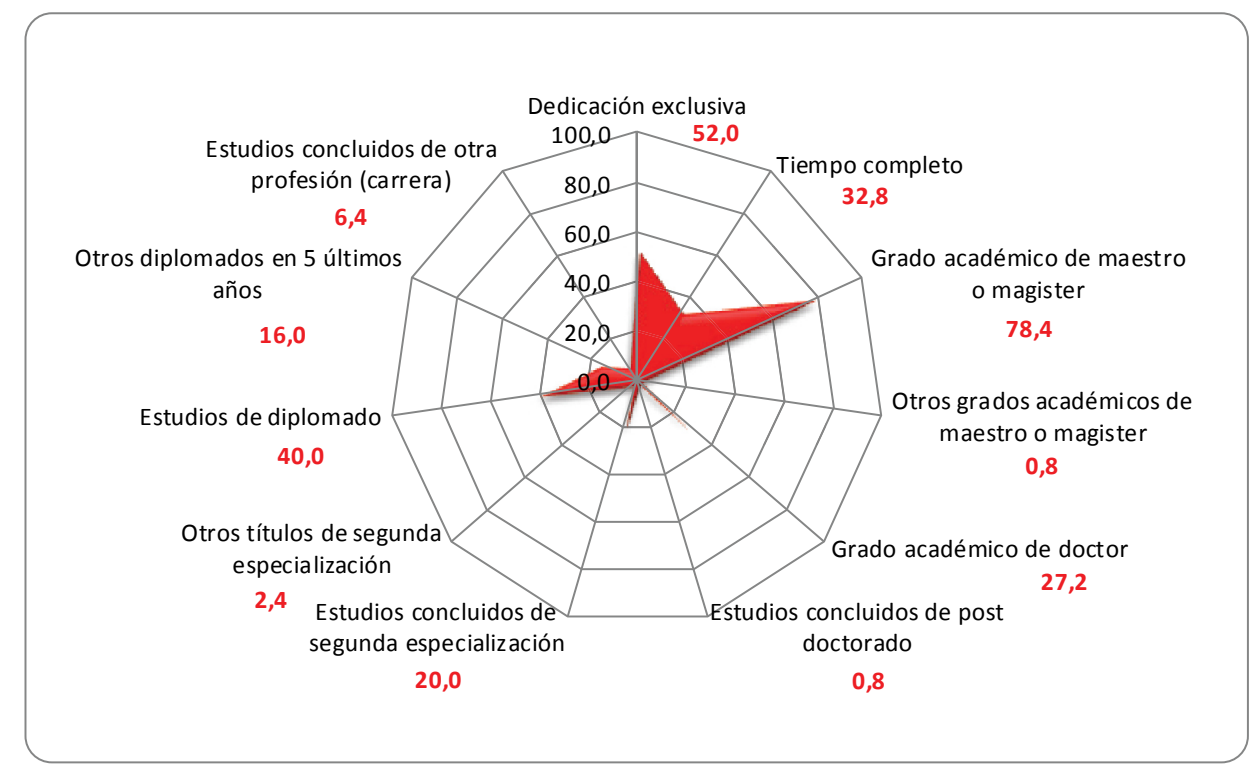

Fuente: Elaboración propia. 
Figura 2. Dispersión de puntos entre las dimensiones de la autoeficacia y el desarrollo profesional de los docentes de la Universidad Nacional Hermilio Valdizán.

Huánuco, 2013.

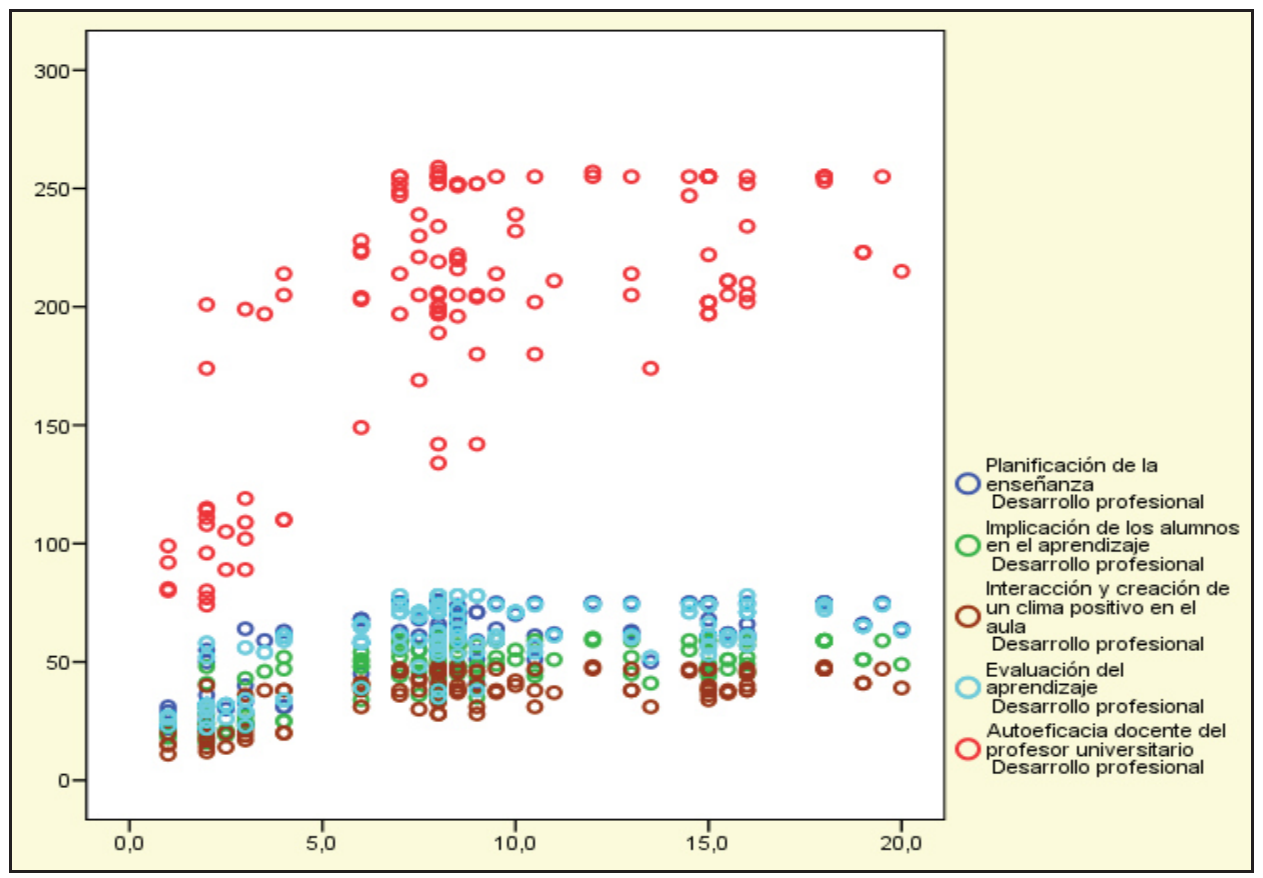

Fuente: Elaboración propia. 